Bull. Korean Math. Soc. 52 (2015), No. 5, pp. 1423-1431

http://dx.doi.org/10.4134/BKMS.2015.52.5.1423

\title{
CLIQUE-TRANSVERSAL SETS IN LINE GRAPHS OF CUBIC GRAPHS AND TRIANGLE-FREE GRAPHS
}

\author{
LiYing Kang AND ERfang Shan
}

\begin{abstract}
A clique-transversal set $D$ of a graph $G$ is a set of vertices of $G$ such that $D$ meets all cliques of $G$. The clique-transversal number is the minimum cardinality of a clique-transversal set in $G$. For every cubic graph with at most two bridges, we first show that it has a perfect matching which contains exactly one edge of each triangle of it; by the result, we determine the exact value of the clique-transversal number of line graph of it. Also, we present a sharp upper bound on the cliquetransversal number of line graph of a cubic graph. Furthermore, we prove that the clique-transversal number of line graph of a triangle-free graph is at most the chromatic number of complement of the triangle-free graph.
\end{abstract}

\section{Introduction}

All graphs considered here are finite, simple and nonempty. For standard terminology not given here we refer the reader to [7].

Let $G=(V, E)$ be a graph with vertex set $V$ and edge set $E$. For a vertex $v \in V$, the open neighborhood $N_{G}(v)$ of $v$ is defined as the set of vertices adjacent to $v$, i.e., $N_{G}(v)=\{u \mid u v \in E\}$. The closed neighborhood of $v$ is $N_{G}[v]=N_{G}(v) \cup\{v\}$. For a subset $S \subseteq V$, the open neighborhood of $S$ is $N_{G}(S)=\bigcup_{v \in S} N(v)$ and the closed neighborhood of $S$ is $N_{G}[S]=\bigcup_{v \in S} N[v]$. The degree of $v$ is equal to $\left|N_{G}(v)\right|$, denoted by $d_{G}(v)$ or simply $d(v)$. A graph is a cubic graph if every vertex has degree 3 . For a subset $S \subseteq V$, the subgraph induced by $S$ is denoted by $G[S]$. As usual, the complete bipartite graph $K_{1,3}$ is called a claw and the complete graph $K_{3}$ a triangle. For a given graph $F$, we say that a graph $G$ is $F$-free if it does not contain $F$ as an induced subgraph. In particular, $K_{1,3}$-free is also called claw-free. $K_{3}$-free is also called Triangle-free. For a family of graphs $\left\{F_{1}, \ldots, F_{k}\right\}$ we say that $G$ is $\left\{F_{1}, \ldots, F_{k}\right\}$-free if it is $F_{i}$-free for all $i=1, \ldots, k$. A bridge is an edge of $G$ whose removal increases

Received November 6, 2013.

2010 Mathematics Subject Classification. 05C69, 05C65, 05 C15.

Key words and phrases. matching, clique-transversal set, clique-transversal number, cubic graph, line graph.

This research was partially supported by the National Nature Science Foundation of China (Nos. 11171207, 11471210). 
the number of components of $G$. The line graph $L(G)$ of $G$ is the graph whose vertices are the edges of $G$, and two vertices of $L(G)$ are connected if and only if the edges corresponding to them share a common vertex in $G$. As is well known, line graphs are a subclass of claw-free graphs.

A matching $M$ in $G$ is a set of pairwise non-adjacent edges; that is, no two edges share a common vertex. If $M$ is a matching, the two ends of each edge of $M$ are said to be matched under $M$, and each vertex incident with an edge of $M$ is said to be covered by $M$. A perfect matching is one which covers every vertex of the graph. A maximum matching is a matching of $G$ that contains the largest possible number of edges. The number of edges in a maximum matching of $G$ is called the matching number of $G$ and denoted $\alpha^{\prime}(G)$. An edge cover of a graph $G$ is defined as a set $S$ of edges of $G$ such that every vertex of $G$ is incident with at least one of members of $S$. The edge cover number, denoted by $\beta^{\prime}(G)$, is the number of edges in a minimum edge cover of $G$.

The clique-transversal set in graphs can be regarded as a special case of the transversal set in hypergraph theory [5]. A clique $C$ of a graph $G$ is a complete subgraph maximal under inclusion and having at least two vertices. According to this definition, isolated vertices are not considered to be cliques here. A clique of order $m$ of $G$ is called an $m$-clique of $G$. A set $D \subseteq V$ is called a clique-transversal set of $G$ if $D$ meets all cliques of $G$, i.e., $D \cap V(C) \neq \emptyset$ for any clique $C$ of $G$. The clique-transversal number, denoted by $\tau_{C}(G)$, is the minimum cardinality of a clique-transversal set of $G$.

Erdös et al. [8] proved that the problem of finding a minimum cliquetransversal set for a graph is NP-hard. It is therefore of interest to determine bounds on the clique-transversal number of a graph. In [8] the authors showed that any graph $G$ of order $n$ has $1 \leq \tau_{C}(G) \leq n-\sqrt{2 n}+3 / 2$ and they observed that $\tau_{C}(G)$ can be very close to $n=|V(G)|$, namely $\tau_{C}=n-o(n)$ can hold. On the basis of the fact above, it is reasonable to ask how drastically $\tau_{C}$ decreases or increases when some assumptions are imposed on the graph $G$. From this point of view, Tuza [14] and Andreae [1] established upper bounds on $\tau_{C}(G)$ for chordal graphs $G$. In [11] we established an upper bound and a sharp lower bound on $\tau_{C}(G)$ for connected $k$-regular graphs $G$. Bacsó and Tuza [4] found a tight upper bound on $\tau_{C}(G)$ for cubic graphs $G$. In [3] authors studied the upper bounds on $\tau_{C}$ for the classes of cographs and clique perfect graphs.

In 1991, Andreae, Schughart and Tuza [2] investigated the clique-transversal numbers for line graphs and complements of line graphs. They obtained the following result.

Theorem 1 (Andreae, Schughart and Tuza [2]). Let $G$ be a connected graph with at least two edges and assume that $G$ is not an odd cycle. Then $\tau_{C}(L(G)) \leq$ $|E(G)| / 2$.

This paper was motivated by the above result, we investigate the extremal behavior of $\tau_{C}(L(G))$ on the line graphs of cubic graphs and triangle-free graphs $G$. For every cubic graph with at most two bridges, we first show that it has 
a perfect matching which contains exactly one edge of each triangle of it; by the result, we can determine the exact value of the clique-transversal number of line graph of it. Furthermore, we present a tight upper bound on $\tau_{C}(G)$ for line graphs of cubic graphs. Finally, we prove that $\tau_{C}(L(G)) \leq \chi(\bar{G})$ for a triangle-free graph $G$ without isolated vertices, and the equality holds if $G$ has minimum degree at least two.

\section{Line graphs of cubic graphs}

For a cubic graph $G$, let $T$ be a triangle of $G$ and $\mid N_{G}(V(T)) \cap(V(G)-$ $V(T)) \mid=3$. If $N_{G}(V(T)) \cap(V(G)-V(T))$ is an independent set of vertices in $G$, we call $T$ an isolated triangle of $G$.

Lemma 2. For every cubic graph $G$ with no isolated triangles, there exists a maximum matching that contains exactly one edge of each triangle in $G$.

Proof. Without loss of generality, we may assume that $G$ is connected. Suppose that $G$ contains no triangles, the assertion is trivial. Otherwise, $G$ contains at least a triangle. Let $M$ be a maximum matching of $G$ that covers triangles of $G$ as many as possible. Note that $M$ contains at most one edge of each triangle in $G$. If $M$ contains exactly one edge of each triangle in $G$, then there is nothing to show. Otherwise, there exists at least a triangle $T=u_{1} u_{2} u_{3}$ whose edges are not in $M$. The following proof is by contradiction.

Let $S=N_{G}(V(T)) \cap(V(G)-V(T))$ and let $S=\left\{x_{1}, x_{2}, x_{3}\right\}\left(x_{i}\right.$ not necessarily distinct), where $x_{i} u_{i} \in E(G)$ for $1 \leq i \leq 3$. If $|S|=1$, i.e., $x_{1}=x_{2}=x_{3}$, then $G$ is isomorphic to $K_{4}$, the assertion immediately follows. If $|S|=2$, without loss of generality, let $x_{1}=x_{2}$. In this case, the induced subgraph $G\left[\left\{x_{1}\right\} \cup V(T)\right]$ is a diamond (the graph $K_{4}-e$ obtained from $K_{4}$ by removing its one edge). By the maximality of $M$ and $E(T) \cap M=\emptyset$, it is easy to see that exactly one of $u_{1} x_{1}, u_{2} x_{1}$ is in $M$ and $u_{3}$ is covered by $M$. Without loss of generality, assume that $x_{1} u_{1} \in M$. Let $M^{\prime}=\left(M \backslash\left\{u_{1} x_{1}\right\}\right) \cup\left\{u_{1} u_{2}\right\}$. Then $M^{\prime}$ is a maximum matching of $G$ that covers more triangles of $G$ than $M$, which contradicts our assumption to $M$. So we may assumes that $|S|=3$, that is, $x_{1}, x_{2}, x_{3}$ are distinct. By the maximality of $M$, there are at least two vertices, say $u_{1}, u_{2}$, of $V(T)$ that are covered by $M$. Thus $u_{1} x_{1}, u_{2} x_{2} \in M$. Suppose that $u_{3}$ is uncovered by $M$. Note that the edge $u_{1} x_{1}$ lies in no triangles of $G$. We remove the edge $u_{1} x_{1}$ from $M$ and add $u_{1} u_{3}$ to $M$. The resulting matching is still maximum and it covers more triangles of $G$ than the primitive $M$, a contradiction. Thus, each vertex of $T$ is covered by $M$, so $u_{i} x_{i} \in M$ for $1 \leq i \leq 3$. Since $G$ contains no isolated triangles, $S$ is not independent. Without loss of generality, assume that $x_{1} x_{2} \in E(G)$. Clearly each $u_{i} x_{i}$ lies in no triangles of $G$. Let $M^{\prime}=\left(M \backslash\left\{u_{1} x_{1}, u_{2} x_{2}\right\}\right) \cup\left\{x_{1} x_{2}, u_{1} u_{2}\right\}$. Then $M^{\prime}$ is a maximum matching of $G$ and it covers more triangles of $G$ than $M$, which is a contradiction. 
Remark. The above assertion is still true when the cubic graph is reduced to a graph with maximum degree at most three.

Lemma $3([7])$. Every cubic graph with at most two bridges has a perfect matching.

Theorem 4. Every cubic graph with at most two bridges has a perfect matching that contains exactly one edge of each triangle in the graph.

Proof. If $G$ contains no isolated triangles, then the result follows from Lemma 2 and Lemma 3. Otherwise, we let $G^{*}$ be the graph obtained from $G$ by contracting each isolated triangle of $G$ to a single vertex. Then $G^{*}$ is a cubic graph with at most two bridges and it contains no isolated triangles. Hence, by Lemmas 2 and $3, G^{*}$ has a perfect matching $M^{*}$ that contains exactly one edge of each triangle in $G^{*}$.

Let $T_{j}=x_{j 1} x_{j 2} x_{j 3}(j=1,2, \ldots, l)$ be all the isolated triangles of $G$. For each $T_{j}=x_{j 1} x_{j 2} x_{j 3}$ of $G$, let $v_{T_{j}}$ be the vertex of $G^{*}$ obtained by contracting the triangle $T_{j}$. Since $M^{*}$ is a perfect matching of $G^{*}$, each $v_{T_{j}}$ is covered by $M^{*}$, and so precisely one edge incident with $v_{T_{j}}$ is in $M^{*}$. Without loss of generality, let $v_{T_{j}} u \in M^{*}$ and $x_{j 1} u \in E(G)$. If $u$ is not the vertex of $G^{*}$ obtained by contracting a triangle of $G$, i.e., $u \neq v_{T_{i}}$ for any $1 \leq i \leq l$, then we remove the edge $v_{T_{j}} u$ from $M^{*}$ and add to $M^{*}$ the edges $x_{j 2} x_{j 3}$ and $x_{j 1} u$. Suppose that $u=v_{T_{k}}$ for some $k \neq j, 1 \leq k \leq l$, i.e., $u$ is obtained by contracting the other triangle $T_{k}$ of $G$. Since $T_{j}$ and $T_{k}$ are isolated, there is exactly one edge between $V\left(T_{j}\right)$ and $V\left(T_{k}\right)$. Let $x_{j 1} x_{k 1} \in E(G)$. Clearly, the edge $x_{j 1} x_{k 1}$ of $G$ corresponds to the edge $v_{T_{j}} v_{T_{k}}$ of $G^{*}$. In $G$, we add the edges $x_{j 1} x_{k 1}, x_{j 2} x_{j 3}$ and $x_{k 2} x_{k 3}$ to $M^{*}$ and remove the edge $v_{T_{j}} v_{T_{k}}$ from $M^{*}$. The updated $M^{*}$ is a set of edges of $G$. Obviously, $M^{*}$ is a perfect matching of $G$ and it contains exactly one edge of each triangle of $G$, the assertion follows.

Remark. The conditions in Lemma 2 and Lemma 3 can not be dropped. Let $B_{1}$ be the graph, called a balloon, obtained from a complete graph $K_{4}$ on four vertices by subdividing an edge of $K_{4}$. Now let $F$ be the cubic graph obtained from disjoint union of three copies of $B_{1}$ and a triangle $C_{3}$ by joining three vertices of the triangle to the vertices of degree 2 in the three copies of $B_{1}$, respectively. By our construction, $F$ is a cubic graph on 18 vertices with a isolated triangle $C_{3}$ and three bridges. It is easily seen that $F$ has a perfecting matching and $\alpha^{\prime}(F)=9$. But there is not a perfecting matching of $F$ that contains edges of the triangle $C_{3}$.

Let $G$ be a connected graph with at least two edges. Clearly, $L(G)$ has just two kinds of cliques, namely the 'star-cliques' and the 'triangle-cliques': the former are the sets $S(x)=\{e \in E(G): e$ is incident with $x\}$, where $x$ is either a vertex of degree $\geq 3$ or a vertex of degree 2 which is not contained in a triangle of $G$, while the latter are the edge sets of the triangles of $G$. Clearly, for any graph $G$ with minimum degree at least two, each clique-transversal set of $L(G)$ must be an edge cover of $G$. 
Theorem 5. For every cubic graph $G$ with at most two bridges, $\tau_{C}(L(G))=$ $\frac{|E(G)|}{3}$.

Proof. Let $D$ be a minimum clique-transversal set of $L(G)$. Then $D$ is also an edge cover of $G$. To cover all vertices of $G$, each edge cover of $G$ contains at least $|V(G)| / 2$ edges of $G$. Thus $\tau_{C}(L(G))=|D| \geq|V(G)| / 2=|E(G)| / 3$. On the other hand, by Theorem 4 , we know that $G$ has a perfect matching $M$ that contains exactly one edge of its each triangle. Hence $M$ is a clique-transversal set of $L(G)$, and thus $\tau_{C}(L(G)) \leq|M|=|E(G)| / 3$.

In [6] a sharp lower bound on the matching number $\alpha^{\prime}(G)$ is obtained for a cubic graph $G$. Further, $\mathrm{O}$ and West [12] found the family $\mathcal{H}_{1}$ of extremal graphs achieving the bound. The family $\mathcal{H}_{1}$ is constructed as follows. Let $B_{1}$ be the graph as mentioned earlier. In fact, this is the smallest graph in which one vertex has degree 2 and the others have degree 3 . Let $T_{1}$ be the family of trees such that every non-leaf vertex has degree 3 and all leaves have the same color in a proper 2-coloring. Let $\mathcal{H}_{1}$ be the family of cubic graphs obtained from trees in $T_{1}$ by identifying each leaf of such a tree with the vertex of degree 2 in a copy of $B_{1}$.

For characterizing the extremal graphs achieving the upper bound, we need the following lemma.

Lemma 6 ([12]). If $G$ is a connected cubic graph on $n$ vertices, then $\alpha^{\prime}(G) \geq$ $(4 n-1) / 9$, and equality holds if and only if $G \in \mathcal{H}_{1}$.

Lemma 7 ([9]). For each graph $G$ of order $n, \beta^{\prime}(G)+\alpha^{\prime}(G)=n$.

Theorem 8. If $G$ is a connected cubic graph of order $n$, then

$$
\frac{|E(G)|}{3} \leq \tau_{C}(L(G)) \leq \frac{10|E(G)|+3}{27} .
$$

Moreover, this left equality holds if and only if $G$ has a perfect matching that contains exactly one edge of each triangle in $G$, while this right equality holds if and only if $G \in \mathcal{H}_{1}$.

Proof. Without loss of generality we may assume that $G$ is connected. Note that each clique-transversal set of $L(G)$ is an edge cover of $G$ while each edge cover of $G$ contains at least $n / 2$ edges of $G$. Hence $\tau_{C}(L(G)) \geq|E(G)| / 3$. This equality holds if and only if $G$ has an edge cover of size $n / 2$ that contains exactly one edge of each triangle in $G$. Obviously, such an edge cover of size $n / 2$ is also a perfecting matching of $G$, as desired.

In order to get the upper bound, we distinguish the following two cases.

Case 1. $G$ contains no isolated triangles.

By Lemma 2, $G$ has a maximum matching that contains exactly one edge of each triangle in $G$. Let $M$ be such a matching. For each vertex uncovered by $M$, take an edge incident with the vertex. Denote by $M_{0}$ the set of all such edges. Then $\left|M_{0}\right|=n-2|M|$. Clearly, $M \cup M_{0}$ is a clique-transversal set of $L(G)$. On 
the other hand, since $\left|M \cup M_{0}\right|=n-|M|=n-\alpha^{\prime}(G), M \cup M_{0}$ is a minimum edge cover of $G$. This implies that $M \cup M_{0}$ is a minimum clique-transversal set of $L(G)$. By Lemma 6, we have

$$
\tau_{C}(L(G))=\left|M \cup M_{0}\right|=n-|M| \leq n-\frac{4 n-1}{9}=\frac{10|E(G)|+3}{27},
$$

the assertion follows.

Case 2. G contains isolated triangles.

As in the proof of Theorem 4, let $G^{*}$ be the graph obtained from $G$ by contracting each isolated triangle of $G$ to a single vertex. Then $G^{*}$ is a connected cubic graph that contains no isolated triangles. Let $S^{*}$ be a minimum clique-transversal set of $L\left(G^{*}\right)$. Then, by Case 1 , we have $\tau_{C}\left(L\left(G^{*}\right)\right) \leq$ $\left(10\left|E\left(G^{*}\right)\right|+3\right) / 27$.

Let $T_{j}(j=1,2, \ldots, l)$ be all the isolated triangles of $G$. Then $\left|E\left(G^{*}\right)\right|=$ $|E(G)|-3 l$. For each $T_{j}$, let $v_{T_{j}}$ be the 'contracting vertex' of $G^{*}$ corresponding to $T_{j}$. Clearly, at least an edge incident with each 'contracting vertex' of $G^{*}$ belongs to $S^{*}$. Let $v_{T_{j}} u \in S^{*}$ and $x_{j} u \in E(G)$ where $x_{j} \in V\left(T_{j}\right)$. If $u \neq v_{T_{i}}$ for any $1 \leq i \leq l$, then we remove the edge $v_{T_{j}} u$ from $S^{*}$ and add to $S^{*}$ the edge $x_{j} u$ and the edge of $T_{j}$ not incident with $x_{j}$. Suppose that $u=v_{T_{k}}$ for some $k \neq j, 1 \leq k \leq l$, i.e., $u$ is obtained by contracting the other triangle $T_{k}$ of $G$. Since $T_{j}$ and $T_{k}$ are isolated, there is exactly one edge between $V\left(T_{j}\right)$ and $V\left(T_{k}\right)$. Let $x_{j} x_{k} \in E(G)$ where $x_{k} \in V\left(T_{k}\right)$. Clearly, the edge $x_{j} x_{k}$ of $G$ corresponds to the edge $v_{T_{j}} v_{T_{k}}$ of $G^{*}$. In $G$, we replace the edge $v_{T_{j}} v_{T_{k}}$ of $S^{*}$ by the edge $x_{j} x_{k}$ and add the edges of $T_{j}$ and $T_{k}$ not incident with $x_{j}$ and $x_{k}$ respectively, to $S^{*}$. The updated $S^{*}$ is a set of edges of $G$. Obviously, $S$ is a clique-transversal set of $L(G)$. Hence,

$$
\begin{aligned}
\tau_{C}(L(G)) \leq|S| \leq\left|S^{*}\right|+l & =\frac{10\left|E\left(G^{*}\right)\right|+3}{27}+l \\
& <\frac{10\left(\left|E\left(G^{*}\right)\right|+3 l\right)+3}{27} \\
& =\frac{10|E(G)|+3}{27} .
\end{aligned}
$$

We next show that $\tau_{C}(L(G))=(10|E(G)|+3) / 27$ if and only if $G \in \mathcal{H}_{1}$ for a connected cubic graph $G$ of order $n$.

Suppose $G \in \mathcal{H}_{1}$. By Lemma $6, \alpha^{\prime}(G)=(4 n-1) / 9$. The vertices set in $L(G)$ corresponding to three edges incident with each vertex in $G$ induces a 3 -clique. The edges set of $G$ corresponding to each clique-transversal set in $L(G)$ is an edge cover set of $G$. According to Lemma 7, we have

$$
\tau_{C}(L(G))=\beta^{\prime}(G)=n-\alpha^{\prime}(G)=n-(4 n-1) / 9=(10|E(G)|+3) / 27 .
$$

Hence $\tau_{C}(L(G))=(10|E(G)|+3) / 27$.

Conversely, suppose that $\tau_{C}(L(G))=(10|E(G)|+3) / 27$ for a connected cubic graph $G$ of order $n$. By the above proof, the Case 2 cannot appear and 
the inequality in equation (1) is equality. Thus, $\alpha^{\prime}(G)=(4 n-1) / 9$. We have $G \in \mathcal{H}_{1}$ by Lemma 6 .

This completes the proof of Theorem 8 .

Now we are ready to present another upper bound on $\tau_{C}(L(G))$ for the line graph $L(G)$ of a cubic graph $G$. Moreover, we also give a characterization of the extremal graphs attaining the upper bound. For this purpose, we define the graph $B$ and the cubic graph $H(g)$ as follows. First, let $B$ be a graph with girth $r$ in which one vertex has degree two and all others have degree 3 and such that the order $g$ of $B$ is as small as possible. For example, if $r=3, B$ is the balloon $B_{1}$ with $g=5$. If $r=4$, then $B$ is the complete bipartite graph $K_{3,3}$ with one edge subdivided, and $g=7$. The existence of $B$ having girth $r$ is shown by observing that an $r$-cage with one edge subdivided will serve (see [13], for a proof that $r$-cages exist for all $r \geq 3)$. Now let $H(g)$ be the cubic graph obtained from disjoint union of three copies of $B$ by adding a new vertex and joining it with the vertex of degree 2 in each copy of $B$, respectively.

Lemma 9 ([10]). If $G$ is a cubic graph of order $n$ with girth $r \geq 3$, then $\alpha^{\prime}(G) \geq\left(\frac{3 g-1}{3 g+1}\right) \frac{n}{2}$, where $g$ is the number of vertices in B. Moreover, the equality holds if and only if $G$ is the graph $H(g)$.

For a cubic graph $G$ with girth $r \geq 4$, By Lemma 9, we can slightly improve the result in Theorem 8 .

Theorem 10. If $G$ is a cubic graph of order $n$ with girth $r \geq 3$, then $\tau_{C}(L(G)$ ) $\leq \frac{g+1}{3 g+1}|E(G)|$, where $g$ is the number of vertices in B. Moreover, the equality holds if and only if $G$ is the graph $H(g)$.

Proof. We may assume $G$ is connected. If $r=3$, then $g=5$. By Theorem 8 , we have

$$
\tau_{C}(L(G)) \leq \frac{10|E(G)|+3}{27} \leq \frac{3}{8}|E(G)|=\frac{g+1}{3 g+1}|E(G)|,
$$

the assertion holds. So we may assume that $r \geq 4$. Since $G$ contains no triangles, all cliques of $L(G)$ are stars of $G$. Let $M$ be a maximum matching of $G$. For each vertex uncovered by $M$, take an edge incident with the vertex. Denote by $M_{0}$ the set of all chosen edges. Then $\left|M_{0}\right|=n-2|M|$. Clearly, $M \cup M_{0}$ is a clique-transversal set of $L(G)$ as well a minimum edge cover of $G$. By Lemma 9, we have

$$
\tau_{C}(L(G))=\left|M \cup M_{0}\right|=n-|M| \leq n-\left(\frac{3 g-1}{3 g+1}\right) \frac{n}{2}=\frac{g+1}{3 g+1}|E(G)| .
$$

That the equality holds in Theorem 10 implies that the equality in Lemma 9 holds. Therefore, the equality holds if and only if $G$ is the graph $H(g)$.

Remark. If $G$ is a cubic graph with girth $r=3$, then $g=5$. By above result, we have $\tau_{C}(L(G)) \leq 3|E(G)| / 8$. In this case, the result in Theorem 8 is better 
than the result $3|E(G)| / 8$. However, when girth $r \geq 4$, since $g \geq 7$, we have $\tau_{C}(L(G)) \leq(g+1)|E(G)| /(3 g+1) \leq 4|E(G)| / 11 \leq(10|E(G)|+3) / 27$.

For general regular graphs, we propose the following problem.

Problem. Find a sharp upper bound on the clique-transversal number for line graphs of $k$-regular graphs, where $k \geq 4$.

\section{Line graphs of triangle-free graphs}

Let $\chi(G)$ denote the chromatic number of a graph $G$. The complement $\bar{G}$ of $G$ is the graph with the same vertex set but whose edge set consists of the edges not present in $G$.

For a general graph $G$ with girth at least 4, we can obtain an upper bound on $\tau_{C}(L(G))$ in terms of $\chi(\bar{G})$.

Theorem 11. For a triangle-free graph $G$ without isolated vertices, $\tau_{C}(L(G))$ $\leq \chi(\bar{G})$; and if $G$ has minimum degree at least two, then the equality holds.

Proof. Since $G$ is triangle-free, the line graph $L(G)$ of $G$ contains only the "star-cliques", so each edge cover of $G$ is a clique-transversal set of $L(G)$. Hence $\tau_{C}(L(G)) \leq \beta^{\prime}(G)$. Note that $\alpha^{\prime}(G)+\beta^{\prime}(G)=|V(G)|$. To obtain our result, it is sufficient to show that $|V(G)|-\alpha^{\prime}(G)=\chi(\bar{G})$. Let $k=\chi(\bar{G})$ and let $\left\{V_{1}, V_{2}, \ldots, V_{k}\right\}$ be the partition of $V(G)$, where $V_{i}$ denotes the set of vertices assigned colour $i$ in a proper $k$-colouring of $\bar{G}$. The triangle-freeness of $G$ implies that $1 \leq\left|V_{i}\right| \leq 2$. Clearly, the set of edges induced by the colour classes $V_{i}$ with $\left|V_{i}\right|=2$ in $G$ is a matching of $G$. So $\alpha^{\prime}(G) \geq|V(G)|-\chi(\bar{G})$, that is, $\chi(\bar{G}) \geq|V(G)|-\alpha^{\prime}(G)$. On the other hand, let $M=\left\{u_{i} v_{i} \mid i=1,2, \ldots, \alpha^{\prime}(G)\right\}$ be a maximum matching of $G$. We colour the vertices of $\bar{G}$ with the colours $1,2, \ldots,|V(G)|-\alpha^{\prime}(G)$ as follows: assign the colours $1,2, \ldots,|V(G)|-2 \alpha^{\prime}(G)$ to each vertex $V(G)-V(M)$ respectively and the colours $|V(G)|-2 \alpha^{\prime}(G)+$ $1, \ldots,|V(G)|-\alpha^{\prime}(G)$ to each pair $\left\{u_{i}, v_{i}\right\}$ respectively. It is easy to see that the colouring is a proper colouring of $\bar{G}$. So $\chi(\bar{G}) \leq|V(G)|-\alpha^{\prime}(G)$. Thus $\chi(\bar{G})=|V(G)|-\alpha^{\prime}(G)$.

If $G$ has minimum degree at least two, then the set of edges incident with any vertex of $G$ induces a clique of $L(G)$. Note that the line graph $L(G)$ of $G$ contains only the "star-cliques". Thus each minimum clique-transversal set of $L(G)$ corresponds to a minimum edge cover of $G$. Hence $\tau_{C}(L(G))=\beta^{\prime}(G)$, that is, $\tau_{C}(L(G))=\chi(\bar{G})$.

\section{References}

[1] T. Andreae, On the clique-transversal number of chordal graphs, Discrete Math. 191 (1998), no. 1-3, 3-11.

[2] T. Andreae, M. Schughart, and Zs. Tuza, Clique-transversal sets of line graphs and complements of line graphs, Discrete Math. 88 (1991), no. 1, 11-20.

[3] S. Aparna Lakshmanan and A. Vijayakumar, The $\langle t\rangle$-property of some classes of graphs, Discrete Math. 309 (2009), no. 1, 259-263. 
[4] G. Bacsó and Zs. Tuza, Clique-transversal sets and weak 2-colorings in graphs of small maximum degree, Discrete Math. Theor. Comput. Sci. 11 (2009), no. 2, 15-24.

[5] C. Berge, Hypergraphs, Amsterdam: North-Holland, 1989.

[6] T. Biedl, E. D. Demaine, C. A. Duncan, R. Fleischer, and S. G. Kobourov, Tight bounds on maximal and maximum matchings, Discrete Math. 285 (2004), no. 1-3, 7-15.

[7] J. A. Bondy and U. S. R. Murty, Graph Theory, Springer, 2008.

[8] P. Erdős, T. Gallai, and Zs. Tuza, Covering the cliques of a graph with vertices, Discrete Math. 108 (1992), no. 1-3, 279-289.

[9] T. Gallai, Über extreme Punkt-und Kantenmengen, Ann. Univ. Sci. Budapest. Eőtvős Sect. Math. 2 (1959), 133-138.

[10] A. M. Hobbs and E. Schmeichel, On the maximum number of independent edges in cubic graphs, Discrete Math. 42 (1982), no. 2-3, 317-320.

[11] E. F. Shan, T. C. E. Cheng, and L. Y. Kang, Bounds on the clique-transversal number of regular graphs, Sci. China Ser. A 51 (2008), no. 5, 851-863.

[12] O. Suil and D. B. West, Balloons, cut-edges, matchings, and total domination in regular graphs of odd degree, J. Graph Theory 64 (2010), no. 2, 116-131.

[13] W. T. Tutte, Connectivity in Graphs, University of Toronto Press, Toronto, 1966.

[14] Zs. Tuza, Covering all cliques of a graph, Discrete Math. 86 (1990), no. 1-3, 117-126.

\section{LIYING KANG}

Department of Mathematics

Shanghai University

Shanghai 200444, P. R. ChinA

E-mail address: lykang@shu.edu.cn

ERFANG SHAN

Department of Mathematics

ShANGHAi UNIVERSITY

Shanghai 200444, P. R. China

AND

School of Management

Shanghai University

Shanghai 200444, P. R. China

E-mail address: efshan@shu.edu.cn 